\title{
Gesture Discrimination in Primary Progressive Aphasia: The Intersection between Gesture and Language Processing Pathways
}

\author{
Natalie Nelissen, ${ }^{1}$ Mariella Pazzaglia, ${ }^{2,3}$ Mathieu Vandenbulcke, ${ }^{4}$ Stefan Sunaert, ${ }^{5}$ Katrien Fannes, ${ }^{1}$ Patrick Dupont, ${ }^{1}$ \\ Salvatore M. Aglioti, ${ }^{2,3}$ and Rik Vandenberghe ${ }^{1,6}$ \\ ${ }^{1}$ Laboratory for Cognitive Neurology, Experimental Neurology Section, Katholieke Universiteit Leuven, 3000 Leuven, Belgium, ${ }^{2}$ Dipartimento di Psicologia, \\ Università degli Studi di Roma "La Sapienza," 00185 Rome, Italy, ${ }^{3}$ Istituto di Ricovero e Cura a Carattere Scientifico, Fondazione Santa Lucia, 00142 Rome, \\ Italy, and Departments of ${ }^{4}$ Psychiatry, ${ }^{5}$ Radiology, and ${ }^{\circ}$ Neurology, University Hospitals Leuven, 3000 Leuven, Belgium
}

The issue of the relationship between language and gesture processing and the partial overlap of their neural representations is of fundamental importance to neurology, psychology, and social sciences. Patients suffering from primary progressive aphasia, a clinical syndrome characterized by comparatively isolated language deficits, may provide direct evidence for anatomical and functional association between specific language deficits and gesture discrimination deficits. A consecutive series of 16 patients with primary progressive aphasia and 16 matched control subjects participated. Our nonverbal gesture discrimination task consisted of 19 trials. In each trial, participants observed three video clips showing the same gesture performed correctly in one clip and incorrectly in the other two. Subjects had to indicate which of the three versions was correct. Language and gesture production were evaluated by means of conventional tasks. All participants underwent high-resolution structural and diffusion tensor magnetic resonance imaging. Ten of the primary progressive aphasia patients showed a significant deficit on the nonverbal gesture discrimination task. A factor analysis revealed that this deficit clustered with gesture imitation, word and pseudoword repetition, and writing-to-dictation. Individual scores on this cluster correlated with volume in the left anterior inferior parietal cortex extending into the posterior superior temporal gyrus. Probabilistic tractography indicated this region comprised the cortical relay station of the indirect pathway connecting the inferior frontal gyrus and the superior temporal cortex. Thus, the left perisylvian temporoparietal area may underpin verbal imitative behavior, gesture imitation, and gesture discrimination indicative of a partly shared neural substrate for language and gesture resonance.

\section{Introduction}

The intimate relationship between language and gesture execution and recognition is currently an intensely debated topic with important ontogenetic (Bates and Dick, 2002) and phylogenetic ramifications (Rizzolatti and Craighero, 2004; Willems and Hagoort, 2007; Toni et al., 2008; Zlatev, 2008). Mounting evidence indicates that even complex aspects of language may be ultimately "grounded" in perception and action (Barsalou, 2008; Fischer and Zwaan, 2008; Gentilucci and Volta, 2008; Nazir et al., 2008).

Most of the clinical-neurological studies of the relationship between language and gesture processing (Liepmann, 1908; Goodglass and Kaplan, 1963; Duffy and Duffy, 1981; Kertesz and Hooper, 1982) predated the availability of neuroimaging techniques necessary for defining the neuroanatomical link with pre-

Received Jan. 19, 2010; revised March 3, 2010; accepted March 25, 2010.

This work was supported by Research Foundation Flanders (FW0) (Grants G.0076.02 and G0668.07 to R.V.) Katholieke Universiteit Leuven (Grants $0 \mathrm{~T} / 08 / 056$ and EF/05/014 to R.V.), and Federaal Wetenschapsbeleid belspo (Grant IUAP P6/29). R.V. is an FW0 senior clinical investigator, and N.N. is an FW0 research fellow.

Correspondence should be addressed to Dr. Rik Vandenberghe, Neurology Department, University Hospitals Leuven, Herestraat 49, 3000 Leuven, Belgium. E-mail: rik.vandenberghe@uz.kuleuven.ac.be.

DOI:10.1523/JNEUROSCI.0321-10.2010

Copyright $\odot 2010$ the authors $\quad 0270-6474 / 10 / 306334-08 \$ 15.00 / 0$ cision. Primary progressive aphasia (PPA), a clinical syndrome characterized by initially relatively isolated language deficits due to progressive neurodegenerative disease (Mesulam, 1982; Mesulam et al., 2003), may represent a unique model for providing direct evidence on the cognitive and neuroanatomical link between gesture discrimination and language. PPA patients show variations in the degree of impairment in motor speech, word comprehension, grammatical processing, and praxis, resulting in subtypes such as the semantic (Hodges et al., 1992), nonfluent/ agrammatic (Grossman, 2002), or logopenic variants (GornoTempini et al., 2004) of PPA. This intersubject heterogeneity across PPA patients lies at the basis of our experimental approach. We examined which language task scores cluster with gesture discrimination scores and how shared zones of brain volume loss can account for this symptom clustering.

From neuroanatomical studies of gesture processing in stroke (De Renzi and Lucchelli, 1988; Maher and Rothi, 2002; Goldenberg and Karnath, 2006), one of the most consistent findings is the association of parietal damage with deficits in imitation of gestures and actual use of tools and objects (Goldenberg, 2009). According to a computed tomography study of a limited number of stroke cases, when both the comprehension and production of gestures are affected, the left supramarginal and angular gyrus are 
Table 1. Demographics, clinical subtypes, and scores of the neuropsychological experimental evaluation

\begin{tabular}{|c|c|c|c|c|c|c|c|c|c|c|c|c|c|c|c|c|c|c|}
\hline & \multicolumn{16}{|l|}{ Cases } & \multirow{2}{*}{$\begin{array}{l}\text { Control subjects } \\
{[\text { mean (SD)] }}\end{array}$} & \multirow[b]{2}{*}{ Tota } \\
\hline & 1 & 2 & 3 & 4 & 5 & 6 & 7 & 8 & 9 & 10 & 11 & 12 & 13 & 14 & 15 & 16 & & \\
\hline Subtype & SemD & SemD & SemD & SemD & SemD & SemD & SemD & SemD & SemD & PNFA & PNFA & PNFA & PNFA & PNFA & PNFA & LPA & & \\
\hline Gender & $\mathrm{M}$ & M & $\mathrm{M}$ & M & $\mathrm{F}$ & M & $\mathrm{F}$ & M & M & M & $\mathrm{F}$ & M & $\mathrm{F}$ & M & M & $\mathrm{F}$ & & \\
\hline Age (yr) & 59 & 72 & 61 & 75 & 61 & 64 & 60 & 72 & 81 & 67 & 71 & 73 & 51 & 76 & 75 & 76 & $68.6(7.6)$ & \\
\hline Education (yr) & 19 & 19 & 12 & 9 & 15 & 20 & 13 & 14 & 12 & 13 & 11 & 12 & 12 & 13 & 10 & 8 & $13.2(4.0)$ & \\
\hline Duration (yr) & 3 & 5 & 8 & 8 & 2 & 7 & 2 & 6 & 2 & 4 & 4 & 3 & 3 & 5 & 7 & 3 & & \\
\hline Gesture discrimination & 9 & 15 & 13 & 14 & 18 & 17 & 12 & 15 & 14 & 10 & 19 & 18 & 17 & 17 & 11 & 13 & $17.9(1.0)$ & 19 \\
\hline Intransitive & 4 & 5 & 6 & 6 & 9 & 7 & 6 & 6 & 5 & 5 & 9 & 8 & 8 & 9 & 4 & 7 & $8.5(0.6)$ & 9 \\
\hline Transitive & 5 & 10 & 7 & 8 & 9 & 10 & 6 & 9 & 10 & 5 & 10 & 10 & 9 & 8 & 7 & 6 & $9.4(0.7)$ & 10 \\
\hline BNT & 36 & 35 & 4 & 3 & 44 & 13 & 25 & 34 & 1 & 6 & 8 & 44 & 49 & 58 & 11 & 36 & $53.9(4.2)$ & 60 \\
\hline $\begin{array}{l}\text { Living-nonliving } \\
\text { PALPA }\end{array}$ & 28 & 30 & 15 & 5 & 52 & 28 & 38 & 43 & 1 & 27 & 6 & 58 & 53 & 57 & 21 & 49 & $55.0(2.6)$ & 60 \\
\hline Auditory WP matching & 38 & 31 & 20 & 19 & 39 & 36 & 35 & 38 & 27 & 23 & 38 & 39 & 38 & 39 & 32 & 40 & $39.9(0.3)$ & 40 \\
\hline Visual WP matching & 35 & 30 & 18 & 20 & 38 & 36 & 36 & 39 & 28 & 25 & 38 & 39 & 39 & 39 & 28 & 40 & $40.0(0.0)$ & 40 \\
\hline Associative-semantic task & 18 & 18 & 18 & 13 & 28 & 26 & 6 & 28 & 15 & 11 & 23 & 30 & 27 & 29 & 15 & 28 & $27.9(1.6)$ & 30 \\
\hline Lexical decision & 83 & 86 & 82 & 75 & 87 & 93 & 76 & 97 & 66 & 78 & 84 & 91 & 91 & 96 & 90 & 96 & $95.9(3.4)$ & 100 \\
\hline Repetition & 55 & 129 & 140 & 111 & 148 & 149 & 133 & 146 & 153 & 28 & 149 & 133 & 14 & 150 & 98 & 148 & $139.5(14.5)$ & 160 \\
\hline Word reading & 75 & 73 & 79 & 79 & 80 & 80 & 46 & 80 & 71 & 63 & 66 & 80 & 4 & 74 & 79 & 80 & $80.0(0.0)$ & 80 \\
\hline Pseudoword reading & 22 & 22 & 24 & 21 & 24 & 24 & 4 & 24 & 21 & 20 & 20 & 23 & 0 & 17 & 23 & 24 & $23.9(0.3)$ & 24 \\
\hline Dictation & 18 & 26 & 28 & 22 & 28 & 29 & 15 & 30 & 22 & 2 & 21 & 25 & 29 & 30 & 13 & 30 & $29.7(0.6)$ & 30 \\
\hline Gesture imititation & 13 & 22 & 22 & 23 & 24 & 24 & 20 & 24 & 23 & 18 & 21 & 24 & 20 & 21 & 19 & 22 & $23.9(0.3)$ & 24 \\
\hline Object use & 20 & 20 & 10 & 10 & 22 & 22 & 15 & 22 & 21 & 7 & 22 & 22 & 21 & 22 & 12 & 18 & $21.9(0.3)$ & 22 \\
\hline
\end{tabular}

Test scores in bold indicate significantly decreased performance compared with the control population (one-sided, $p<0.05$ ). Gesture discrimination, Gesture discrimination test; $\mathrm{M}$, male; F, female; living-nonliving, living-nonliving confrontation naming test; WP, word-picture; SemD, semantic dementia.

involved, while pure production deficits rely relatively more on more anterior regions (Heilman et al., 1982; Heilman and Gonzalez-Rothi, 1993). This led to the classic model of "visuokinesthetic motor engrams," which guide the sequencing and timing of motor movements, that are localized to these inferior parietal regions (Heilman et al., 1982; Heilman and GonzalezRothi, 1993). A more recent voxel-based lesion-symptom mapping study in apraxic patients without aphasia suggested that the left inferior frontal gyrus rather than inferior parietal cortex is associated with deficits in gesture discrimination (Pazzaglia et al., 2008). Two lesion subtraction studies implicated a relatively extensive region containing inferior frontal as well inferior parietal cortex in gesture recognition (Tranel et al., 2003; Buxbaum et al., 2005).

The aim of the current study was not to define the gesture discrimination network per se but to determine the overlap between gesture discrimination and language networks. We combined state-of-the-art imaging techniques with extensive behavioral analysis and an ad hoc designed gesture discrimination task. This approach yielded direct, novel evidence on where language and gesture processing pathways intersect.

\section{Materials and Methods}

Subjects

A consecutive series of 16 patients ( 11 men, 5 women) fulfilling the diagnostic criteria for PPA (Mesulam, 2001, 2003; Mesulam et al., 2003) were recruited from the academic Memory Clinic of the University Hospitals Leuven. The average age was 68.4 years (SD, 8.2 years; range, $51-81$ years), mean educational level was 13.3 years (SD, 3.5 years; range, $8-20$ years), and mean disease duration was 4.5 years (SD, 2.1 years; range, $2-8$ years) (Table 1). All subjects were strictly right-handed [Edinburgh handedness score of 100 (Oldfield, 1971)] except for case 8 [handedness score of -43 (Oldfield, 1971)]. Subjects were recruited only if their disease stage still allowed them to fully comprehend and follow the task instructions of our behavioral protocol. Part of this cohort (cases 1, 3, 4, 10, 11, and 14-16) overlapped with the participants of a previous PPA study (Vandenbulcke et al., 2005). Based on detailed longitudinal clinical-neurological evaluation, clinical neuropsychological assessment, and state-of-the-art clinical neuroimaging findings [magnetic resonance imaging (MRI) in all cases; fluorodeoxyglucose-positron emission tomography in 13 cases], and before any experimental procedure, nine patients were assigned by consensus to a semantic dementia subtype and six patients to a progressive nonfluent aphasia (PNFA) subtype (Neary et al., 1998). One subject was assigned to the more recently defined logopenic aphasia (LPA) subtype (Gorno-Tempini et al., 2004).

A control group of 16 cognitively intact subjects (11 men, 5 women), matched for age (mean, 68.6 years; SD, 7.6 years; range, $55-81$ years), educational level (mean, 13.2 years; SD, 4.0 years; range, $8-22$ years), and handedness (Edinburgh handedness score +100 in 15 control subjects and -73 in 1 subject) underwent the same protocol. Control subjects were recruited via an advertisement in a regional newspaper asking for volunteers above the age of 50 years for participation in a scientific study. Among the exclusion criteria were subjective memory or other cognitive complaints, a history of significant neurological or psychiatric illness, or lesions on brain MRI.

After complete description of the study, written informed consent was obtained in accordance with the Declaration of Helsinki. The study protocol was approved by the Ethics Committee of the University Hospitals Leuven.

\section{Experimental task}

Subjects were seated in front of a 19 inch computer screen at a distance of $\sim 50 \mathrm{~cm}$ and viewed video clips in which a male actor performed intransitive (not involving the use of objects, e.g., waving goodbye; $n=9$ ) and transitive (involving the use of objects, e.g., playing guitar; $n=10$ ) meaningful gestures. Each gesture could be performed either correctly (target) or incorrectly (distractor). For both intransitive and transitive gestures, two types of distractors were used. For intransitive gestures, distractors were created by asking the actor to perform a given gesture (e.g., hitchhiking) either by changing the hand or finger configuration crucial for correctly identifying the gesture (e.g., hitchhiking performed by extending the little finger instead of the thumb) or by changing the spatial position of the involved body part (e.g., hitchhiking performed with hand above the head) (for another example of an intransitive gesture, see Fig. $1 A-C)$. For transitive gestures, distractors were created by exchanging the object in a given correct gesture (e.g., playing guitar) with an object that was inappropriate for that particular action. The inappropriate object could be semantically related (e.g., flute) or unrelated (e.g., broom) to the correct object (e.g., guitar) (Fig. 1D-F). Fifty-seven of the sixty video clips used in a previous study of gesture discrimination in 


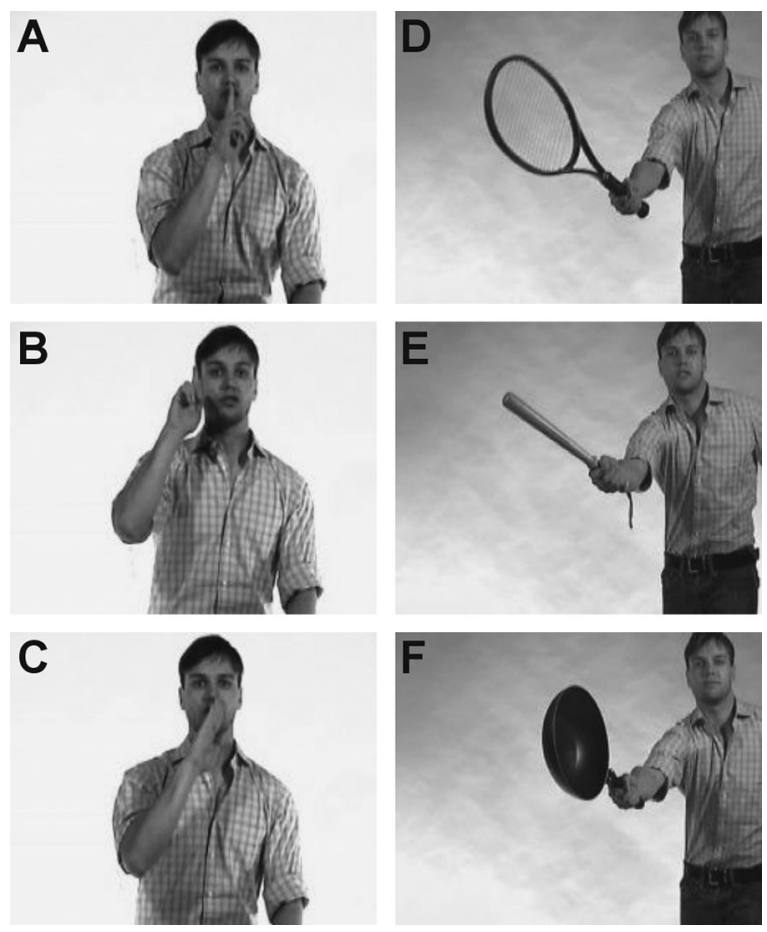

Figure 1. Static images from a representative trial. $\boldsymbol{A}-\boldsymbol{C}$, Intransitive gestures. $\mathbf{D}-\boldsymbol{F}$, Transitive gestures. For each trial, a correct version of a gesture is shown together with two distractors. Each trial consisted of one correctly executed gesture $(\boldsymbol{A}, \boldsymbol{D})$ and two distractors, shown in random order. For intransitive gestures, distractors consisted of an incorrect spatial configuration of either the proximal $(\boldsymbol{B})$ or distal parts $(\boldsymbol{C})$ of the involved limb. As distractors for transitive gestures, the correct object was replaced with an inappropriate object that had either a high $(\boldsymbol{E})$ or low $(\boldsymbol{F})$ degree of semantic similarity with the correct object.

stroke patients (Pazzaglia et al., 2008) were used in the present research. The clips not used in the present study depicted an intransitive, specifically Italian gesture.

Subjects underwent 19 trials in total. Each trial consisted of the presentation of a video clip of a correctly executed gesture (target) and two incorrectly executed versions (distractors) of the gesture (Fig. 1). These three video clips each had a duration of $6 \mathrm{~s}$ and were presented sequentially, with the first clip in the upper third of the screen, followed $4 \mathrm{~s}$ after the end of the first clip by a second clip in the middle third of the screen, and, finally, by a third clip after another $4 \mathrm{~s}$ in the lower third of the screen. As soon as a clip ended, a static image of the most representative frame appeared and remained on the screen until all three clips had been shown and the subject had selected one of the three versions. The location and order of appearance of the video clips within each trial and the order of trials were randomized for every subject. The subject was instructed to indicate which was the correctly executed version of the gesture either by pointing to the target or by verbal responses. No feedback was provided during the task. Each correct response scored 1 point. Before the experimental task, subjects received instructions as well as a test trial to ensure that each of them had fully understood the task.

\section{Neuropsychological battery}

Per protocol, patients and control subjects also received an extensive neuropsychological assessment using a priori selected conventional tasks. Confrontation naming was assessed by means of the Boston Naming Test (BNT) (Kaplan et al., 1983; Mariën et al., 1998) and by means of a confrontation naming test developed to detect category effects and which we will refer to as the "living-nonliving naming test" (Laiacona and Capitani, 2001; Vandenbulcke et al., 2006). We assessed single word comprehension and repetition, and reading of words and pseudowords by means of a selection of tests from the Psycholinguistic Assessment of Language Processing in Aphasia (PALPA) (Kay et al., 1992; Bastiaanse et al., 1995). In the word-picture matching task, a concrete noun was pre- sented auditorily (PALPA item 45) or visually (PALPA item 46) together with a target picture and four distractors. Two distractors were semantically related to the target, a third was perceptually similar, and the fourth picture was unrelated. Subjects had to point to the target. In the associative-semantic task (PALPA item 49), a noun was presented visually together with four-choice noun stimuli (a target noun, a noun that was semantically related to the target, and two unrelated nouns), and subjects had to underline or circle the noun that matched the sample stimulus most closely in meaning, for a total of 15 word series with high imageability and 15 with low imageability. During the lexical decision task (PALPA item 24), 80 words and 80 pseudowords were presented visually in random order. Subjects had to press a left- or right-hand key depending on the lexical status of the stimulus. In the auditory word repetition task (PALPA item 9), the examiner, who was sitting in front of the patient, pronounced 80 nouns and 80 pseudowords, which the subject had to repeat. In the word reading task, subjects had to read aloud 80 words (PALPA item 30) and 24 pseudowords (PALPA item 35). Writingto-dictation was tested by means of a subtest of the Aachen Aphasie Test, in which subjects had to write seven words and three sentences from dictation (Graets et al., 1992). We also evaluated the imitation of 12 intransitive arm and hand gestures (cross; salute; wave; hitchhiking; hunger; finger click; crazy; strangling; extension of digits 1-3 with flexion of thumb and digit 4; opposition of index and thumb; extension thumb and digit 4 with flexion of digits 1-3; and index finger rubbing the nose). An examiner demonstrated the gesture, and the test subject had to reproduce the gesture immediately thereafter.

We also evaluated manual use of 11 actual objects (comb, scissors, eraser, matches, whistle, stethoscope, tooth brush, sugar tongues, mouth organ, screwdriver, and telephone). We placed an object on the table in front of the subject and instructed the subject to display how the object is used. Subjects were allowed to manipulate the object. For both the gesture imitation and object use tests, correctly executed gestures received a score of 2 , gestures that were recognizable but only partially adequate received a score of 1 point, and no points were awarded for any other type of response (De Renzi et al., 1980). Performance was videotaped and stored.

\section{Image acquisition}

Patients and control subjects received their MR scan within 3 months of the neuropsychological testing. A 3 tesla Philips Achieva system using a dedicated head coil and SENSE parallel imaging provided T1-weighted images using a three-dimensional turbo field echo sequence [repetition time $(\mathrm{TR})=9.6 \mathrm{~ms}$; echo time $(\mathrm{TE})=4.6 \mathrm{~ms}$; field of view $(\mathrm{FOV})=$ $250 \times 250 \mathrm{~mm}^{2}$; 182 slices; voxel size, $1.2 \times 0.98 \times 0.98 \mathrm{~mm}^{3}$ ]. Diffusion tensor images were obtained in a sagittal plane using a spin-echo echoplanar imaging sequence $(\mathrm{TR}=11 \mathrm{~s} ; \mathrm{TE}=55 \mathrm{~ms}$; FOV $=220 \times 220$ $\mathrm{mm}^{2}$; 68 slices; voxel size, $1.7 \times 1.7 \times 2.2 \mathrm{~mm}^{3}$ ). Diffusion-weighted images ( $\mathrm{b}$ value $=800 \mathrm{~s} / \mathrm{mm}^{2}$ ) were acquired along 45 noncollinear directions. One set of images was acquired with no diffusion weighting ( $\mathrm{b}$ value $=0 \mathrm{~s} / \mathrm{mm}^{2}$ ).

\section{Primary analysis}

Behavioral data. We analyzed the gesture discrimination scores by means of a two-factor repeated-measures ANOVA, with gesture type (two levels: transitive vs intransitive) as the within-subjects factor and group (two levels: PPA vs control subjects) as the between-subjects factor. We also determined which of the 16 individual PPA patients had a pathological score compared with the control group using a one-tailed modified $t$ test (Crawford and Howell, 1998).

To determine which language variables were linked most closely with gesture discrimination and to reduce the number of variables for input into the subsequent voxel-based morphometry (VBM) analysis, we conducted a factor analysis of the neuropsychological tests of all the subjects $(n=32)$ (Statistica 6.0, StatSoft). Factor analysis is a method used for detecting the relational structure between variables. The aim of this analysis is to find underlying unobserved variables (factors) that explain the variability among the observed data. The factor loading of a test can be interpreted as the correlation between that test and the underlying factor. The amount of variability within the observed data that can be accounted 
for by a factor is given by its eigenvalue. Factors were consecutively extracted and orthogonalized to each other. Only factors with an eigenvalue $>1$ were retained (Kaiser criterion). A variance maximizing (vari$\max$ ) rotation of the original variable space was used to obtain an interpretable pattern of factor loadings while preserving the factor orthogonality. Factor scores for every subject and factor were derived from these factor loadings and indicate how well a subject performs on the neuropsychological tests clustered by a given factor. The factor analysis procedure was identical to that used in previous studies (Vandenbulcke et al., 2005; Molenberghs et al., 2009).

Voxel-based morphometry. An optimized VBM analysis was performed on the T1-weighted image using SPM5 (Wellcome Trust Centre for Neuroimaging, http://www.fil.ion.ucl.ac.uk/). This fully automatic procedure involves iterative segmentation into different tissue classes based on voxel intensity and prior probability, bias field correction for intensity inhomogeneities in the MR image, and registration of the resulting gray matter (GM) maps to the tissue probability maps in Montreal Neurological Institute (MNI) space. The final GM maps were modulated to compensate for the volumetric differences introduced by the spatial normalization. An erosion/dilatation procedure allowed for removing most of the extracerebral voxels misclassified as GM. Normalized modulated GM maps $(2 \times 2 \times 2$ $\mathrm{mm}^{3}$ voxels) were smoothed with an isotropic Gaussian kernel with a full width at half-maximum of $8 \mathrm{~mm}$.

Individual factor scores of the PPA patients were entered as independent variables in a voxelwise multiple linear regression analysis with GM volume as the dependent variable. The threshold for significance was set at an uncorrected voxel-level $p$ value of $<0.001$ in combination with a cluster-level $p$ value of $<0.05$ corrected for the whole brain volume. Cluster extent inference was adjusted for nonstationary residual smoothness (Hayasaka et al., 2004) using the VBM5 toolbox (http://dbm.neuro. uni-jena.de/vbm/download/).

Probabilistic tractography. For the clusters of significant brain volume loss we defined the structural connectivity by means of probabilistic tractography in the healthy control subjects. Diffusion tensor imaging (DTI) data were analyzed using FSL 4.0 (FMRIB Software Library, http:// www.fmrib.ox.ac.uk/fsl/index.html). After correction for eddy currents and head motion, the diffusion tensor was fitted to the diffusion data. A probabilistic fiber-tracking method was used (Behrens et al., 2003). First, the uncertainty on the diffusion parameters was estimated by local probability density functions built up by running Markov chain Monte Carlo sampling. These density functions were then used to estimate the probability of the existence of a connection through the data field. An extension of this method (Behrens et al., 2007) allows one to model multiple fiber orientations per voxel. In our study, we used the default setting of modeling at most two (crossing) fibers within each voxel. Seed masks for probabilistic tractography were significant clusters obtained from the VBM analysis performed in this study (as defined earlier in this article). Each cluster was binarized and slightly dilated (using a sphere with a radius of $4 \mathrm{~mm}$ ). The dilation step ensures that the seed mask extends sufficiently into the white matter to begin tracking. The b0 image was coregistered to the skull-stripped, T1-weighted anatomical MR, which was in turn warped to the MNI T1 template. The parameters from these two spatial alignments were concatenated and then inversed to obtain the transformation from MNI standard space to the individual subject's native DTI space. FSL 4.0 uses these transformations as the fiber tracking itself is always performed in native space, whereas the input seeds and resulting fiber tracts were in MNI standard space in our study. Fiber tract images of individual subjects were thresholded to include all voxels through which at least one streamline from the seed mask had passed. These thresholded maps were summed across subjects, resulting in a composite map where each voxel value represents the percentage of subjects showing evidence of a tract. Tracts that could be recovered in $>75 \%$ of the control subjects are shown in Figure 4 . These analyses were conducted for the patients and the normal control group separately.

\section{Secondary analysis}

In the patient group, we performed a multiple linear regression analysis with gesture discrimination score as the dependent variable, age and
Table 2. The correlation of the neuropsychological test with the factors 1, 2, and 3

\begin{tabular}{lccc}
\hline & Factor & \\
\cline { 2 - 4 } & 1 & 2 & 3 \\
\hline Eigenvalue & 7.61 & 2.41 & 1.08 \\
Variance explained & $58.5 \%$ & $18.6 \%$ & $8.3 \%$ \\
Gesture discrimination & 0.53 & 0.03 & $\mathbf{0 . 7 5}$ \\
BNT & $\mathbf{0 . 9 2}$ & 0.05 & 0.18 \\
Living-nonliving naming & $\mathbf{0 . 9 1}$ & 0.04 & 0.14 \\
Auditory word-picture matching & $\mathbf{0 . 9 2}$ & 0.02 & 0.19 \\
Visual word-picture matching & $\mathbf{0 . 9 1}$ & -0.04 & 0.25 \\
Associative-semantic task & $\mathbf{0 . 7 8}$ & 0.22 & 0.44 \\
Lexical decision & $\mathbf{0 . 8 3}$ & 0.28 & 0.18 \\
Repetition & 0.06 & 0.50 & $\mathbf{0 . 7 2}$ \\
Word reading & 0.04 & $\mathbf{0 . 9 7}$ & 0.16 \\
Pseudoword reading & 0.08 & $\mathbf{0 . 9 6}$ & 0.14 \\
Writing-to-dictation & 0.55 & 0.15 & $\mathbf{0 . 6 9}$ \\
Imitation & 0.21 & 0.28 & $\mathbf{0 . 8 5}$ \\
Object use & $\mathbf{0 . 7 2}$ & 0.01 & 0.50 \\
\hline
\end{tabular}

Tests that contribute most to a given factor are indicated in bold.

disease duration as independent continuous variables, and PPA subtype (SemD, PNFA, and LPA) as the independent dummy variable.

In regions that showed a significant correlation between volume and individual scores on a given factor, we determined within the PPA group the Pearson correlation coefficients between the raw scores of each of the tests that loaded on that factor $(r>0.70)$ and the regional gray matter volume. As outliers can drive such correlation analyses disproportionately, we removed outliers from these analyses using the conventional criterion of a Cooke's distance $>1$.

\section{Results}

\section{Behavioral analysis}

The main effect of group was significant $\left(F_{(1,30)}=18,3, p<\right.$ $0.0005)$, but the main effect of gesture type $\left(F_{(1,30)}=2,3, p=\right.$ $0.14)$ and the interaction effect between group and gesture type did not reach significance $\left(F_{(1,30)}=2,4, p=0.13\right)$. Patients obtained significantly lower gesture discrimination scores compared with control subjects (mean score: $14.6 / 19$ vs $17.9 / 19$, respectively) (Table 1). Subjects performed similarly on intransitive gestures compared with transitive gestures (mean score: PPA patients, 72.2 vs $80.6 \%$, respectively; control subjects, 94.4 vs $94.4 \%$, respectively). At an individual level, 10 of the PPA patients had a significantly lower score on the gesture discrimination task (one-sided, $p<0.05$ ) than control subjects (Crawford and Howell, 1998) (Table 1). The patients' performance in the gesture discrimination task could not be explained by age, disease duration, or PPA subtype (multiple linear regression, $p>0.55$ ).

\section{Correlations with VBM}

Factor analysis extracted three factors, together explaining $85.4 \%$ of the total variance observed (Table 2). The first factor predominantly clustered confrontation naming, auditory and visual word-picture matching, the associative-semantic task, lexical decision, and object use (Table 2). The second factor grouped word and pseudoword reading (Table 2). The third factor clustered gesture discrimination, word repetition, gesture imitation, and writing-to-dictation (Table 2).

Individual scores for the factor with the highest loadings for gesture discrimination (factor 3 , Table 2) correlated significantly and positively with gray matter volume in the left supramarginal gyrus extending into the posterior part of the superior temporal gyrus [MNI coordinates: $-64,-50$, and $16 ; Z=3.92 ; 236$ voxels; corrected (corr.) cluster-level inference, $p=0.036$ ] (Fig. 2A). The mean gray matter volume in this cluster correlated significantly with gesture discrimination score $(r=0.80, p=0.0002)$ 
(Fig. $2 B)$, gesture imitation $(r=0.56, p=$ $0.025)$ (Fig. $2 C)$, dictation $(r=0.80, p=$ $0.0002)$, and repetition of words $(r=0.77$, $p=0.0007)$ and pseudowords $(r=0.70$, $p=0.0035$ ) (Fig. 2D). Statistically, the repetition scores in case 13 were considered an outlier and were not included in the correlational analysis (Cook's distance, 2.68). None of the other correlations between mean GM volume and neuropsychological test scores described above suffered from outliers (Cook's distance, $<0.67)$. Gray matter volume in this left perisylvian temporoparietal cluster correlated both with intransitive $(r=$ $0.61, p<0.05)$ and transitive gesture discrimination scores $(r=0.73, p<0.005)$. The correlation between gray matter volume and gesture discrimination scores in this region was significant in both semantic dementia $(r=0.77, p<0.05)$ and PNFA $(r=0.85, p<0.05)$ patients.

Individual scores for the factor with the highest loadings for naming and comprehension (factor 1, Table 2) correlated significantly with decreased gray matter volume in the left anterior temporal cortex (MNI coordinates: $-48,8$, and -20 , $Z=4.92 ; 3320$ voxels; corr. cluster-level inference, $p<0.001$ ) (Fig. $3 A$ ). The mean gray matter volume in this cluster correlated with confrontation naming scores [BNT: $r=0.93, p=2 \times 10^{-7}$ (Fig. 3C); living-nonliving confrontation naming test (Laiacona and Capitani, 2001): $r=0.85$, $\left.p=2 \times 10^{-5}\right)$; auditory and visual word-picture matching $(r=$ $0.73, p=0.0015$ and $r=0.71, p=0.0023$, respectively) (Fig. $3 D$ ); visual lexical decision $(r=0.70, p=0.0024)$; the associativesemantic test $(r=0.65, p=0.006)$; and object use $(r=0.54, p=$ $0.031)]$. The correlation with gesture discrimination scores (Fig. $3 B)$ did not reach significance $(r=0.3673, p=0.16)$.

Individual scores for the factor that clustered mainly reading scores (Table 2) did not correlate with gray matter volume in any region of the brain at our preset threshold.

\section{Probabilistic tractography}

The left perisylvian temporoparietal cluster (Fig. 2) was connected anteriorly with the left inferior frontal operculum and posteriorly with the superior and middle temporal gyrus. This pattern was present in at least $75 \%$ of control subjects as well as in at least $75 \%$ of PPA patients (Fig. 4).

\section{Discussion}

As a group, PPA patients showed a significant gesture discrimination deficit. This deficit was closely associated with gesture imitation deficits and with deficits in verbal tasks requiring imitative behaviors such as repetition of words and pseudowords and writing-todictation (Table 2; Fig. 2). This symptom cluster correlated with volume loss in the left perisylvian parietotemporal junction (Fig. 2). Another cluster of tasks consisted of confrontation naming, word comprehension, lexical decision, associative semantics of single words, tool use, and, more weakly, gesture discrimination. This cluster correlated with anterior temporal volume loss, in line with earlier studies (Hodges et al., 1992; Gorno-Tempini et al., 2004;
B

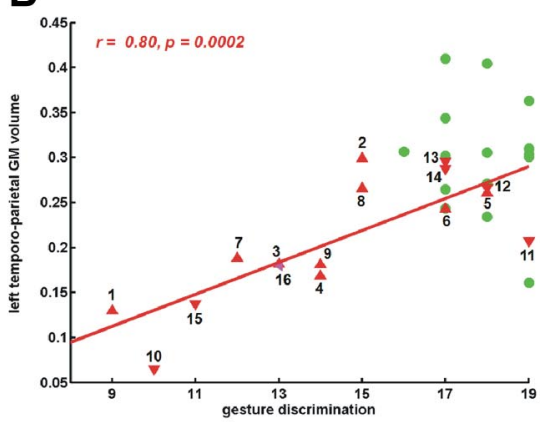

D

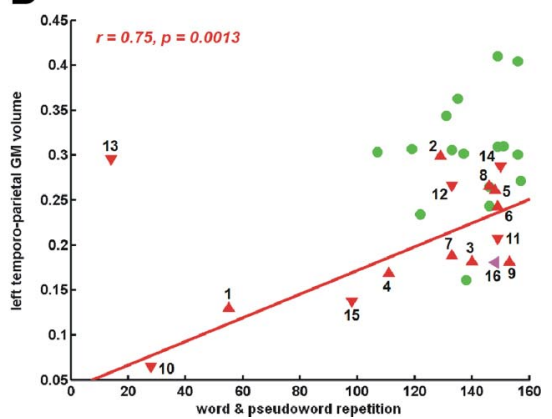

Figure 2. Correlation of gray matter volume with factor 3. A, Voxel-based partial correlation of GM volume with scores on factor this case was removed from the linear regression analysis. Case numbers refer to Table 1. Green, Control subjects; upward red triangle, semantic dementia; downward red triangle, progressive nonfluent aphasia; pink arrow, logopenic aphasia.

Grossman et al., 2004) and demonstrating the face validity of our approach.

Contrary to our a priori hypothesis based on a previous stroke study using the same videos (Pazzaglia et al., 2008), we did not find any correlations between inferior frontal cortex and gesture discrimination scores. First of all, it is important to note that while we did not find the inferior frontal region in our localizationist VBM approach, DTI analysis revealed that the inferior parietal region that we obtained was connected with the inferior frontal cortex (Fig. 4). The negative finding in inferior frontal cortex may be related to methodological differences between the previous and the current study. The exact mode of presentation of videos differed between the two studies: in the stroke study (Pazzaglia et al., 2008), each trial consisted of a single video of a gesture. Correct and incorrect versions of the same gesture were intermingled with videos of other gestures. Subjects had to give a yes/no response to each gesture. In our version, the three versions of the same gesture (the target and the two distractors) were presented within the same trial. Subjects were required to select the correct gesture from among the three versions. When a video is shown in isolation, the subjects need to infer which gesture is intended. When three videos of the same gesture are shown within the same trial, the presentation of the intended gesture will help them reject the distractors. As a second methodological difference, in the current experiment a static image of the most representative fragment remained on the screen at the end of each video so that short-term memory load was lower than when only the video was presented. Presentation of the different versions of a given gesture across trials (Pazzaglia et al., 2008) may invoke working memory processes that may rely relatively more on left inferior frontal cortex. These differences in task and stimulus 
A
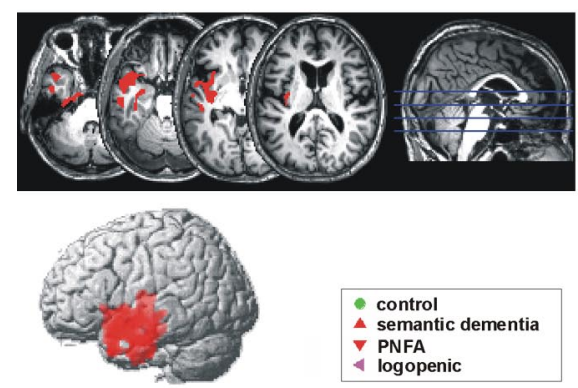

C.

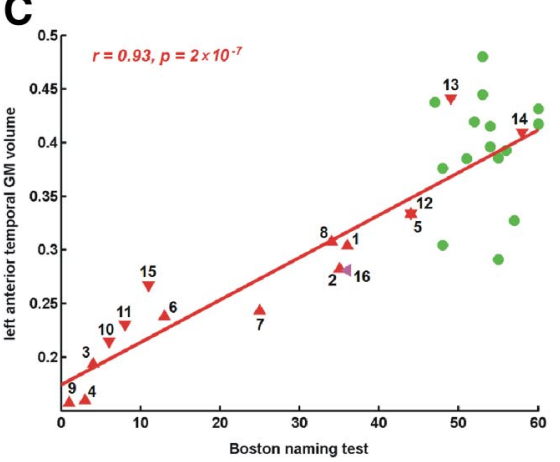

B
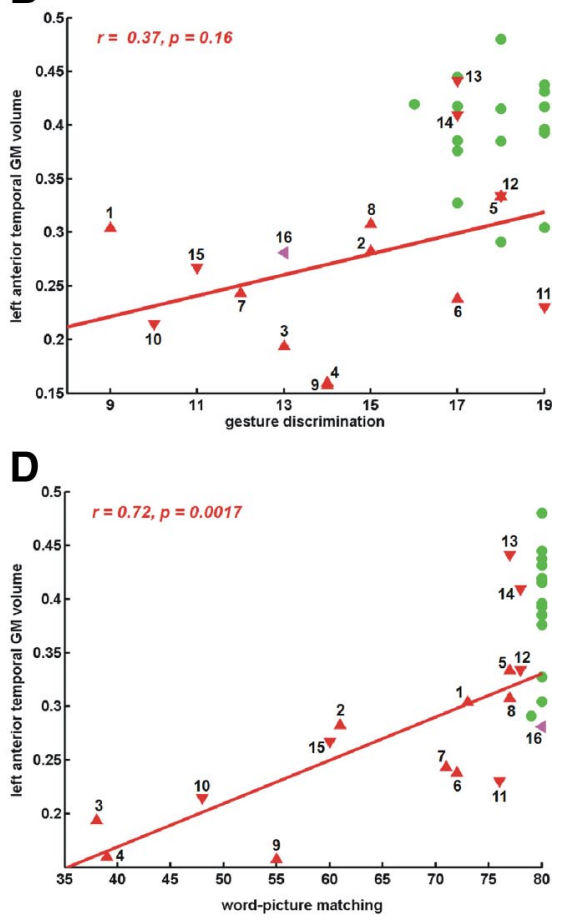

Figure 3. Correlation of GM volume with the lexical-semantic factor. $\boldsymbol{A}$, Voxel-based correlation of $\mathrm{GM}$ volume with scores on factor 1 (voxel-level uncorrected, $p<0.001$; cluster-level corrected, $p<0.05$ ). $\boldsymbol{B}$, Correlation of mean gray matter volume in this cluster with gesture discrimination scores. $\boldsymbol{C}$, Correlation of mean GM volume in this cluster with Boston Naming Test scores. $D$, Correlation of mean gray matter volume in this cluster with word-picture matching scores. Case numbers refer to Table 1. See Figure 2 for other information.

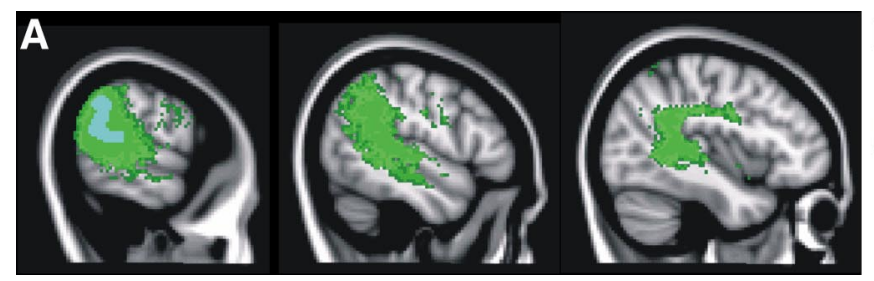

Figure 4. Probabilistic fiber tracking with the temporoparietal cluster as seed region (shown in light blue). $\boldsymbol{A}, \mathrm{A}$ random control subject (control 1, voxels are shown only when at least 500 streamlines from the seed region pass through the voxel). $B$, Composite maps showing voxels present in at least $75 \%$ (yellow/blue) of control subjects.

presentation may have changed the balance between inferior frontal and inferior parietal involvement. The sensitivity of VBM is also very much dependent on the composition of the study group and the composition of the neuropsychological assessment that is entered into the factor analysis.

Our main analysis was pooled across different PPA variants. Behaviorally, in a secondary analysis we did not find a significant difference in gesture discrimination scores between clinically defined subgroups. If we analyzed the semantic dementia and PNFA groups separately, the inferior parietal results remained significant in each group. Group studies comparing semantic dementia, PNFA, and LPA reveal neuroanatomical differences (Gorno-Tempini et al., 2004; Grossman et al., 2004), but at the individual level phenotypic, neuropathological, and genetic overlap exists between the clinical subtypes (Mesulam et al., 2008), and even more so if the evolution of symptoms over time is taken into account (Kertesz et al., 2005). The heterogeneity within the PPA group lies at the base of our experimental approach. It allows us to define symptom clusters, a wider range in individ-

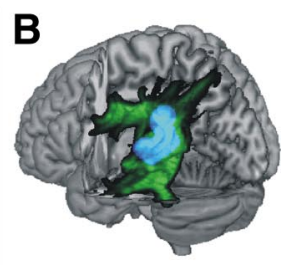

ual factor scores augments the sensitivity of the correlational voxel-based morphometry analysis, and neuroanatomical dissociations between symptom clusters enhance the specificity of the experimental findings.

Our main analysis was pooled across different types of gestures, transitive or intransitive. If we analyzed transitive and intransitive gestures separately, similar results were obtained from our VBM. Since the inferior parietal result was confirmed for the intransitive gestures, it cannot be attributed to object recognition deficits alone or to the use of tools (Lewis, 2006).

The gesture discrimination task invokes many different processes. Subjects perceive the different body parts of the actor and how these parts interact and form a global gesture. Subjects have to match the visually presented gesture with mnemonic representations of the intended gesture and how it is typically executed. Our experimental question was not to outline the neuroanatomy of gesture discrimination per se but to determine which nodes are shared between the language processing and the gesture discrimination pathways. To that end, we identified the language tests that clustered with the gesture discrimination task. Thus, the composition of the cluster provides important clues for the common cognitive processes accounting for the latent variable. Gesture discrimination loaded most heavily on a factor that also included gesture imitation, word or pseudoword repetition, and writing-to-dictation. At first sight, these different functions are disparate. Which perceptual and cognitive processes could be shared by these tasks? Considering the mirror neuron theory (Rizzolatti and Craighero, 2004), the common factor between these tasks may be the "resonance" component. Motor resonance mechanisms refer to the automatic selection of action primitives on which imitation and judgments regarding action appropriateness are based (Rizzolatti and Craighero, 2004). Word and pseudoword repetition also heavily rely on the circle of parity between perception and action, and the same may hold for writing-to-dictation across modalities (auditory to visual). Individual scores for this factor correlated with volume in a left perisylvian parietotemporal region (Fig. $2 B-D$ ). At a neuronal level, the same populations may mediate a process that is common to the word and the gesture processing tasks. Alternatively, this region may contain neuronal populations that are closely juxtaposed or intermingled, with each fulfilling differential roles in word and gesture processing. The cluster had both an inferior parietal and a posterior temporal component (Fig. $2 A)$. This area was connected anteriorly to the frontal operculum and posteriorly to the superior temporal gyrus and sulcus (Fig. 4). The same or a nearby region has been implicated in conduction aphasia, an aphasia syndrome characterized by deficient repetition of words, in particular pseudowords and semantically un- 
constrained word sequences, with preserved comprehension (Goodglass and Kaplan, 1983; Damasio and Damasio, 2000; Blumstein, 2001). The connectivity pattern of the inferior parietal region closely matches the pattern of "Geschwind's territory" (Catani et al., 2005), which is the relay station of the indirect pathway between posterior temporal and inferior frontal cortex. The key novel finding from our study is that the same perisylvian temporoparietal area is critically important for gesture imitation and discrimination and for word repetition. In monkeys, a circuit composed of the ventral premotor cortex (F5) and parietal area PF is involved in the parity between implicit recognition and execution, and constitutes an observation/execution matching system (Zlatev, 2008). PF serves as the relay between F5 and superior temporal sulcus (Rizzolatti and Luppino, 2001). Clearly, direct studies of the homology between humans and monkeys would be needed to test the homology between the perisylvian temporoparietal area we found and area PF.

According to the dual-stream model of the functional anatomy of language (Hickok and Poeppel, 2007), the dorsal pathway, to which the left perisylvian temporoparietal junction belongs, is mainly involved in auditory-motor integration acting as a sensorimotor interface between a phonological network (posterior superior temporal sulcus) and an articulatory network (posterior inferior frontal gyrus, premotor cortex, and anterior insula). According to this model the posterior dorsal-most part of the temporal lobe and parietal operculum are involved in translating acoustic speech signals into articulatory representations (Hickok and Poeppel, 2007). Our findings indicate that a similar principle may be true for gesture processing and that, given the resolution of current MRI techniques, the same or a nearby region may serve as an interface linking gesture recognition and gesture production. The ventral pathway, with the anterior temporal pole as a key node, is mainly involved in sound identification ("what," speech recognition) (Hickok and Poeppel, 2007). The contribution of the left anterior temporal pole in language and semantic processing has been highlighted by studies of semantic dementia relating the semantic processing deficit to neurodegeneration of the anterior temporal pole (Hodges et al., 1992; Rogers and McClelland, 2004). Loss of knowledge of the purpose and prototypical use of tools and objects has been described in semantic dementia patients and is one example of the nonverbal deficits that may occur in such patients (Hodges et al., 2000). It probably also accounts for the correlation of gesture discrimination with measures of semantic processing in our sample (Table 1, Table 2, column 1).

To conclude, the left perisylvian parietotemporal area subserves a generic and critical role in the circle of parity between perception and production of both words and gestures.

\section{References}

Barsalou LW (2008) Grounded cognition. Annu Rev Psychol 59:617-645.

Bastiaanse R, Bosje M, Visch-BrinkE (1995) Psycholinguïstische testbatterij voor de taalverwerking van Afasiepatiënten (PALPA). Hove, UK: Lawrence Erlbaum Associates.

Bates E, Dick F (2002) Language, gesture, and the developing brain. Dev Psychobiol 40:293-310.

Behrens TE, Woolrich MW, Jenkinson M, Johansen-Berg H, Nunes RG, Clare S, Matthews PM, Brady JM, Smith SM (2003) Characterization and propagagion of uncertainty in diffusion-weighted MR images. Magn Reson Med 50:1077-1088.

Behrens TE, Berg HJ, Jbabdi S, Rushworth MF, Woolrich MW (2007) Probabilistic diffusion tractography with multiple fibre orientations: what can we gain? Neuroimage 34:144-155.

Blumstein S (2001) Deficits of speech production and speech perception in aphasia. In: Handbook of neuropsychology, Vol 3 (Berndt RS, ed), pp 95-113. Amsterdam: Elsevier Science.

Buxbaum LJ, Kyle KM, Menon R (2005) On beyond mirror neurons: internal representations subserving imitation and recognition of skilled object-related actions in humans. Brain Res Cogn Brain Res 25:226-239.

Catani M, Jones DK, ffytche DH (2005) Perisylvian language networks of the human brain. Ann Neurol 57:8-16.

Crawford J, Howell D (1998) Comparing an individual's test score against norms derived from small samples. Clin Neuropsychol 12:482-486.

Damasio A, Damasio H (2000) Aphasia and the neural basis of language. In: Principles of behavioral and cognitive neurology (Mesulam M, ed), pp 294-315. Oxford, UK: Oxford UP.

De Renzi E, Lucchelli F (1988) Ideational apraxia. Brain 111:1173-1185.

De Renzi E, Motti F, Nichelli P (1980) Imitating gestures. a quantitative approach to ideomotor apraxia. Arch Neurol 37:6-10.

Duffy RJ, Duffy JR (1981) Three studies of deficits in pantomimic expression and pantomimic recognition in aphasia. J Speech Hear Res 24:70-84.

Fischer MH, Zwaan RA (2008) Embodied language: a review of the role of the motor system in language comprehension. Q J Exp Psychol (Colchester) 61:825-850.

Gentilucci M, Volta RD (2008) Spoken language and arm gestures are controlled by the same motor control system. Q J Exp Psychol (Colchester) 61:944-957.

Goldenberg G (2009) Apraxia and the parietal lobes. Neuropsychologia 47:1449-1459.

Goldenberg G, Karnath HO (2006) The neural basis of imitation is body part specific. J Neurosci 26:6282-6287.

Goodglass H, Kaplan E (1963) Disturbance of gesture and pantomime in aphasia. Brain 86:703-720

Goodglass H, Kaplan E (1983) The assessment of aphasia and related disorders. Philadelphia: Lea and Febiger.

Gorno-Tempini ML, Dronkers NF, Rankin KP, Ogar JM, Phengrasamy L, Rosen HJ, Johnson JK, Weiner MW, Miller BL (2004) Cognition and anatomy in three variants of primary progressive aphasia. Ann Neurol 55:335-346.

Graets P, DeBleser R, Willmes K (1992) Akense afasie test. Lisse, The Netherlands: Swets \& Zeitlinger.

Grossman M (2002) Progressive aphasic syndromes: clinical and theoretical advances. Curr Opin Neurol 15:409-413.

Grossman M, McMillan C, Moore P, Ding L, Glosser G, Work M, Gee J (2004) What's in a name: voxel-based morphometric analyses of MRI and naming difficulty in Alzheimer's disease, frontotemporal dementia and corticobasal degeneration. Brain 127:628-649.

Hayasaka S, Phan KL, Liberzon I, Worsley KJ, Nichols TE (2004) Nonstationary cluster-size inference with random field and permutation methods. Neuroimage 22:676-687.

Heilman K, Gonzalez-Rothi L (1993) Apraxia. In: Clinical neuropsychology (Heilman K, Valenstein E, eds), pp 141-163. New York: Oxford UP.

Heilman KM, Rothi LJ, Valenstein E (1982) Two forms of ideomotor apraxia. Neurology 32:342-346.

Hickok G, Poeppel D (2007) The cortical organization of speech processing. Nat Rev Neurosci 8:393-402.

Hodges JR, Patterson K, Oxbury S, Funnell E (1992) Semantic dementia: progressive fluent aphasia with temporal lobe atrophy. Brain 115:1783-1806.

Hodges JR, Bozeat S, Lambon Ralph MA, Patterson K, Spatt J (2000) The role of conceptual knowledge in object use-evidence from semantic dementia. Brain 123:1913-1925.

Kaplan E, Goodglass H, Weintraub S (1983) The Boston naming test. Philadelphia: Lea and Febiger.

Kay J, Lesser R, Coltheart M (1992) Psycholinguistic assessment of language processing in aphasia. Hove, UK: Lawrence Erlbaum Associates.

Kertesz A, Hooper P (1982) Praxis and language: the extent and variety of apraxia in aphasia. Neuropsychologia 20:275-286.

Kertesz A, McMonagle P, Blair M, Davidson W, Munoz DG (2005) The evolution and pathology of frontotemporal dementia. Brain 128:1996-2005.

Laiacona M, Capitani E (2001) A case of prevailing deficit of nonliving categories or a case of prevailing sparing of living categories. Cogn Neuropsychol 18:39-70.

Lewis JW (2006) Cortical networks related to human use of tools. Neuroscientist 12:211-231.

Liepmann H (1908) Drei aufsatze aus dem apraxiegebiet. Berlin: Karger. 
Maher L, Rothi L (2002) Disorders of skilled movement. In: Handbook of neuropsychology, Vol 3, Language and aphasia (Berndt R, ed), pp 269283. Amsterdam: Elsevier Science.

Mariën P, Mampaey E, Vervaet A, Saerens J, De Deyn PP (1998) Normative data for the Boston naming test in native Dutch-speaking Belgian elderly. Brain Lang 65:447-467.

Mesulam M, Wicklund A, Johnson N, Rogalski E, Léger GC, Rademaker A, Weintraub S, Bigio EH (2008) Alzheimer and frontotemporal pathology in subsets of primary progressive aphasia. Ann Neurol 63:709-719.

Mesulam MM (1982) Slowly progressive aphasia without generalized dementia. Ann Neurol 11:592-598.

Mesulam MM (2001) Primary progressive aphasia. Ann Neurol 49:425-432.

Mesulam MM (2003) Current concepts: primary progressive aphasia: a language-based dementia. N Engl J Med 349:1535-1542.

Mesulam MM, Grossman M, Hillis A, Kertesz A, Weintraub S (2003) The core and halo of primary progressive aphasia and semantic dementia. Ann Neurol 54:S11-S14.

Molenberghs P, Gillebert CR, Schoofs H, Dupont P, Peeters R, Vandenberghe R (2009) Lesion neuroanatomy of the sustained attention to response task. Neuropsychologia 47:2866-2875.

Nazir TA, Jeannerod M, Hauk O (2008) The role of sensory-motor systems for language understanding. Forward. J Physiol Paris 102:1-3.

Neary D, Snowden JS, Gustafson L, Passant U, Stuss D, Black S, Freedman M, Kertesz A, Robert PH, Albert M, Boone K, Miller BL, Cummings J, Benson DF (1998) Frontotemporal lobar degeneration: a consensus on clinical diagnostic criteria. Neurology 51:1546-1554.
Oldfield RC (1971) The assessment and analysis of handedness: the Edinburgh inventory. Neuropsychologia 9:97-113.

Pazzaglia M, Smania N, Corato E, Aglioti SM (2008) Neural underpinnings of gesture discrimination in patients with limb apraxia. J Neurosci 28:3030-3041.

Rizzolatti G, Craighero L (2004) The mirror-neuron system. Annu Rev Neurosci 27:169-192.

Rizzolatti G, Luppino G (2001) The cortical motor system. Neuron 31: 889-901.

Rogers T, McClelland J (2004) Semantic cognition. A parallel distributed approach. Cambridge, MA: MIT.

Toni I, de Lange FP, Noordzij ML, Hagoort P (2008) Language beyond action. J Physiol Paris 102:71-79.

Tranel D, Kemmerer D, Adolphs R, Damasio H, Damasio A (2003) Neural correlates of conceptual knowledge for actions. Cogn Neuropsychol 20:409-432.

Vandenbulcke M, Peeters R, Van Hecke P, Vandenberghe R (2005) Anterior temporal laterality in primary progressive aphasia shifts to the right. Ann Neurol 58:362-370.

Vandenbulcke M, Peeters R, Fannes K, Vandenberghe R (2006) Knowledge of visual attributes in the right hemisphere. Nat Neurosci 9:964-970.

Willems RM, Hagoort P (2007) Neural evidence for the interplay between language, gesture, and action: a review. Brain Lang 101:278-289.

Zlatev J (2008) From proto-mimesis to language: evidence from primatology and social neuroscience. J Physiol Paris 102:137-151. 